\title{
Differential trace conditioning to temporal compounds
}

\author{
ROBERT KOSIBA and FRANK A. LOGAN \\ University of New Mexico, Albuquerque, New Mexico 87131
}

\begin{abstract}
Rats were reinforced for responding following the presentation of a light-tone temporal compound, each component having a .5-sec duration separated by a .5-sec empty interval. For different groups, nonreinforced presentations of the compound in the reverse order and either or both of the components separately were included in each session. Results indicated a progressive increase in difficulty as the number of nonreinforced events increased, with behavior being strongly affected by the first component of the compound. Nevertheless, differential performance under the most demanding conditions demonstrate the rat's ability to acquire a temporal compound discrimination, which suggests an interpretation based on interacting stimulus traces.
\end{abstract}

The procedure of compound conditioning has become of increasing interest, particularly in the context of stimulus control of behavior and, more generally, notions concerning attention. The great majority of previous studies (see, e.g., Mackintosh, 1974) involved the simultaneous presentation of two stimuli associated with some schedule of reinforcement followed by testing for control by the elements separately. Among the phenomena revealed by such studies are overshadowing (greater control by one of the elements than the other) and compounding (response to the compound but not to either element separately). The purpose of the present study was to extend this line of research into temporal compounds, i.e., when the two stimuli are not perfectly contiguous in time.

There are several studies in the literature involving a form of temporal compounds (Wickens, Bonn, \& Wickens, 1963; Woodbury, 1943), the most relevant of which was reported by Egger and Miller (1962) in the context of the information hypothesis of secondary reinforcement. In their experimental procedure, the CS compound was composed of two stimuli, with the onset of the second being slightly delayed. The results indicated that only the first stimulus acquired secondary reinforcing properties, which they interpreted to be a result of the fact that the second stimulus was redundant (conveyed no new information). This interpretation was supported by the results of another procedure that involved differential conditioning. Interspersed among trials with the compound CS were trials with the first element presented separately and not followed by pri-

Based on a thesis submitted by the first author to the faculty of the Department of Psychology, University of New Mexico, in candidacy for the MA degree, 1974. Requests for reprints should be sent to the second author at that address, Albuquerque, New Mexico 87131. This research was supported in part by a grant from the National Science Foundation to the second author. mary reinforcement. With this procedure, the second stimulus was informative and did acquire secondary reinforcing properties.

The stimuli in the Egger and Miller study overlapped and were coterminus, but we felt that a more demanding type of temporal compound conditioning would involve differential responding with respect to traces of tempo. rally discrete stimuli. Accordingly, we shall use the symbol S1-S2 to indicate a compound in which S1 first occurs briefly, followed by a brief interval without stimulation, and in tum followed by a brief presentation of S2. Since this research was done in a discriminated operant conditioning context, it was necessary to impose a discrete-trial DRL contingency (Logan, 1961) in an effort to insure that the subjects be exposed to the complete sequence of programmed stimuli.

Because this was basically exploratory research, it seems appropriate to describe the various conditions to be reported in an order which reflects their probable difficulty to the organism. In all conditions, the S1-S2 compound received $100 \%$ reinforcement; the conditions differed with respect to the other stimulus events that occurred and were not the occasion for reinforcement. Consider first the interspersed nonreinforced presentation of S2 alone during conditioning. According to any theory of discrimination learning, this condition should be more difficult than a basic conditioning involving only $\mathrm{S} 1$ and $\mathrm{S} 2$ alone. The presence of $\mathbf{S} 2$ on all trials should increase the similarity of the stimulus events as a result of the explicitly overlapping stimulus elements. Further interest in this condition centers on subsequent testing with S1 alone. A pure attention theory would predict equivalent responding to $\mathrm{S} 1$ alone as to the training compound $\mathrm{S} 1-\mathrm{S} 2$ because extinction to $\mathrm{S} 2$ would focus attention entirely upon S1. The same prediction follows from modified continuity theory (e.g., Wagner, 1969) because repeated decrements in signal value would accrue to $S 2$, thereby enabling $S 1$ 
ultimately to garner all of the available strength. Only a theory emphasizing compounding would predict a lower response level to $S 1$ than to the $S 1-S 2$ compound, because $\mathrm{S} 1$ is only a part of the original configuration from which a generalization decrement would be expected.

Let us next consider the combination of S1-S2 and S2-S1, with the latter nonreinforced. This condition appears to require a discrimination based on the nature of the temporal compound, since both elements occur in both compounds. Contemporary theories of discrimination learning are hard pressed to cope with this condition, since it cannot be solved by selectively attending to one or the other stimulus and nor are the elements of the temporal compound differentially reinforced. Rather, some version of Hull's (1943) notion of stimulus interaction appears to be required. The assumption would be that $\mathrm{S} 1$ in the presence of the immediate trace of $S 2$ is somehow different from $\mathrm{S} 1$ in the absence of that trace, and conversely. This means that responding could be controlled entirely by the first stimulus in the temporal compound. Evidence bearing on this type of interpretation should be revealed by subsequently testing with $\mathrm{S} 1$ and $\mathrm{S} 2$ separately.

The next condition effectively precludes even this basis for solution by including, in addition to $\mathrm{S} 1-\mathrm{S} 2$ and S2-S1, nonreinforced trials with $\mathrm{S} 1$ alone. The complexity is even more apparent in the final condition to be considered, namely $S 1-S 2, S 2-S 1, S 1$, and $S 2$ with only the first of these reinforced. Neither of these last two conditions can be solved on the basis of the first stimulus alone, even granting stimulus interaction. At least as presently conceived, these conditions require temporal compounding. That is to say, only the trace of $\mathrm{S} 1$ as modified by the trace of the subsequent $\mathrm{S} 2$ receives reinforcement, while any other stimulus event, taken singly or in combination, is nonreinforced.

To a large extent, our interest was simply in whether rats could successfully cope with the last of these conditions and hence display reasonably convincing evidence of selective control by a temporal compound. Also of interest was the role of such compounding in the presumably simpler conditions as might be observed by testing elements separately, as well as the selective control that might be gained by the elements of a temporal compound.

\section{METHOD}

\section{Subjects}

Forty-eight female albino rats, 90 days old at the start of the experiment, were obtained from the animal colony in the Department of Psychology at the University of New Mexico. They were maintained on a daily diet of $12 \mathrm{~g}$ Purina Lab Chow with water freely available in their individual home cages.

\section{Apparatus}

Four operant conditioning chambers, programmed for delivery of $45-\mathrm{mg}$ Noyes reinforcement pellets, were employed.
Table 1

Summary of the Experimental Conditions

\begin{tabular}{ccl}
\hline Group & Reinforced & Nonreinforced \\
\hline I & S1 & S2 \\
II & S1 & S2 (DRL) \\
III & S1-S2 & S2 \\
IV & S1-S2 & S2-S1 \\
V & S1-S2 & S2-S1, S1 \\
VI & S1-S2 & S2-S1, S1, S2 \\
\hline
\end{tabular}

Note-The duration of $S 1$ and $S 2$ was .5 sec separated by a. 5 -sec empty interval. For all conditions except I, a 1.5-sec DRL requirement was imposed with a limited hold of $5 \mathrm{sec}$.

The light was provided by a GE $182928-\mathrm{V}$ bulb mounted behind a plastic protector, and the tone was $8,000 \mathrm{~Hz}$ at $90 \mathrm{~dB}$ provided by a Hewlett-Packard Model 200B tone generator. All stimulus events were programmed by a Friden tape reader which "tuned in" to each box in rotation. All timing and related circuitry were performed by electromechanical units, and responses were recorded on counters.

The sequence of events in any one box ran the following time course: (1) Barpressing was monitored for $5 \mathrm{sec}$ and, should a response occur during this time, the trial was skipped and not recovered. (2) The programmed stimulus event was initiated with a .5 sec light or tone, followed by a .5 -sec empty interval and finally a .5 -sec interval that was either empty or contained the other stimulus on compound trials. Responses at any time during these intervals of time immediately terminated the trial without reinforcement. (3) Thereafter, $5 \mathrm{sec}$ were allowed for a response to occur, the tape reader advancing at the end of that time or with a response, whichever came first. Accordingly, the first response that occurred when a box was tuned in terminated that trial, with reinforcement being given only for responses occurring within the 5 -sec interval after the positive stimulus had been completely presented. If no response occurred, a box remained tuned in for $12.5 \mathrm{sec}$; the ITI varied depending on the response latency of the other three subjects.

\section{Procedure}

All rats were given magazine training, handshaping, and continuous reinforcement training for 3 days, after which they were randomly divided into six groups of eight rats each. Four rats of each group were assigned to the counterbalancing conditions, S1 being the tone and S2 being the light for half the subjects and the reverse for the remaining half. The six conditions studied are summarized in Table 1.

A daily session consisted of 100 trials (potential stimulus presentations), half of which were the $S+$ configuration and the other half of which were divided equally among the appropriate $\mathrm{S}$ - configurations. After each group appeared to have reached an asymptotic level of performance, 10 probe trials for each single component not presented during acquisition were administered to Groups III, IV, and V. These test trials were randomly interspersed among the regular trials during the last session of the experiment for that group. As a result of this procedure, the number of sessions differed for the various groups, but this confounding seemed preferable to varying the amount of overtraining before testing for generalization to the elements of the temporal compound.

\section{RESULTS}

Presumably because responses during the first $5 \mathrm{sec}$ during which a box was tuned in resulted in a net loss of opportunities for reinforcement, such responses dropped virtually to zero by Day 4 and hence do not 
enter into any further computations. A discrimination index was computed for each animal for each day by the simple difference between the percentage of responses to St and the percentage of responses to the S-. Since by the 6th day of training all animals in all groups were responding at over $90 \%$ of the trials to the S+ presentations, this discrimination index reflects predominantly the inhibition of responding to $\mathrm{S}-$. The discrimination indices were analyzed as a mixed design analysis of variance, two factors between groups (Discrimination Conditions and Counterbalancing) and one factor within group (Days). The effect of Discrimination Conditions involving all groups over Days 11, 12, and 13 was significant, $F(5,36)=20.77, p<.001$, with the Tukey B test indicating that all of the differences between the sets of groups (I, II, and III) and (V and VI) were significant at the .05 level. An analysis over Days 18, 19, and 20 involving Groups IV, V, and VI revealed a significant effect of Discrimination Conditions, $\mathrm{F}(2,36)=4.95, \mathrm{p}<.025$, with the differences between Group IV and Groups V and VI significant at the .05 level. Since there was no significant effect due to counterbalancing, the two subgroups for each condition were combined and their performance is displayed in Figure 1.

The figure indicates that the DRL requirement for Group II slightly reduced discriminative performance early in training as compared with Group I, but this deficit was recovered such that the two groups'achieved near-perfect performance in about 10 days. As expected, Group III, for whom the positive stimulus was S1-S2 with $\mathrm{S} 2$ alone nonreinforced, showed a more gradual improvement but was not significantly different from Groups I and II by the time their training was terminated. It is instructive at this point to refer to the top two panels of Figure 2, which shows the performance on the test probe trials with $\mathrm{S} 1$ alone for Group III. It is clear that the presence of $\mathbf{S} 2$ in the temporal compound had no effect on the probability of responding to S1 alone.

Group IV, which had $\mathrm{S} 1-\mathrm{S} 2+$ and $\mathrm{S} 2-\mathrm{S} 1-$, was definitely retarded in acquiring the discrimination, although they ultimately performed about as well as the preceding groups. Turning again to Figure 2 , it can be seen that this level of performance resulted from essentially complete control by the first element of the reinforced compound. Very little responding was occasioned by the second element of the compound which, of course, was also the first element of the negative compound.

As expected, Groups V and VI displayed the greatest difficulty in developing discriminative behavior, but their ultimate performance was nevertheless noteworthy. A discrimination index of 70 could arise, for example, from $95 \%$ responding to $S+$ and $25 \%$ responding to $S-$, which is clearly compelling evidence of temporal compounding. The principal source of difficulty for the rats in these conditions can be gleaned from the bottom panels of Figure 2, where it can be seen that even nonreinforced training with the first element of the compound separately did not totally eliminate responding to that stimulus event. Actually, it is the difference between the level of responding to the first element alone and that to the compound which provides the strictest evidence for selective control by the compound itself.

\section{DISCUSSION}

The results of this study generally conformed to the rather informal expectations described in the introduc-

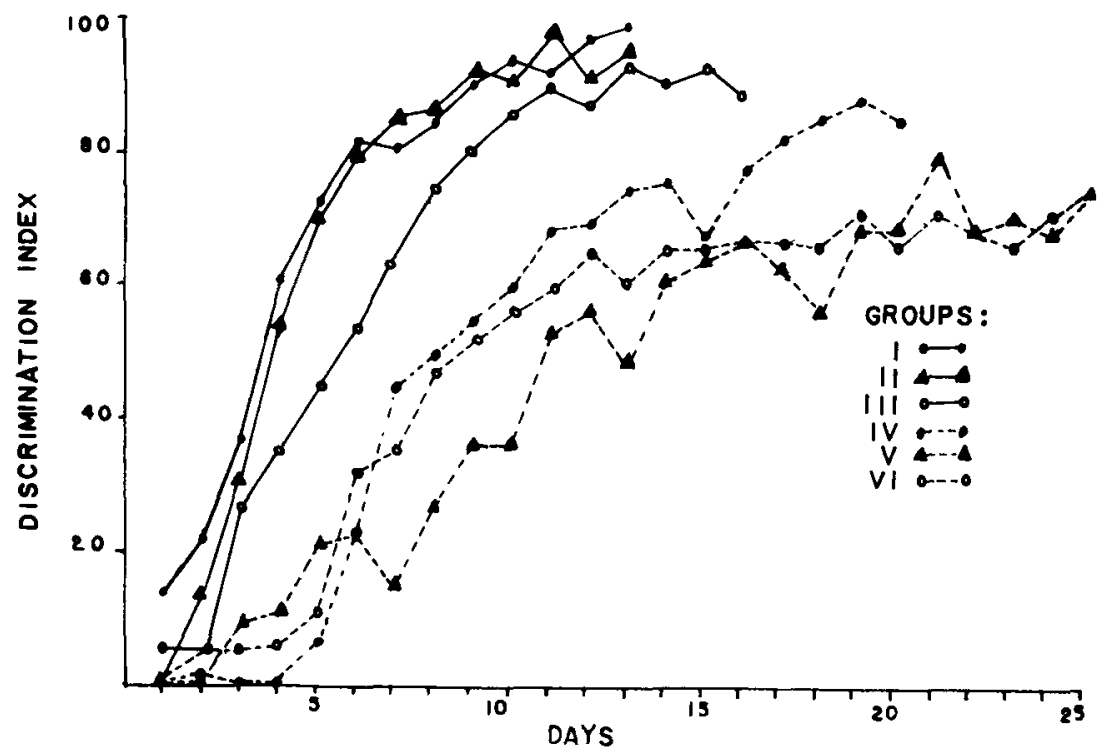

Figure 1. Discrimination index (percent response to $S+$ minus percent response to $S_{-}$) for the various groups over days of training. See Table 1 . The curves combine the counterbalancing of tone and light as $S 1$ and $S 2$. 

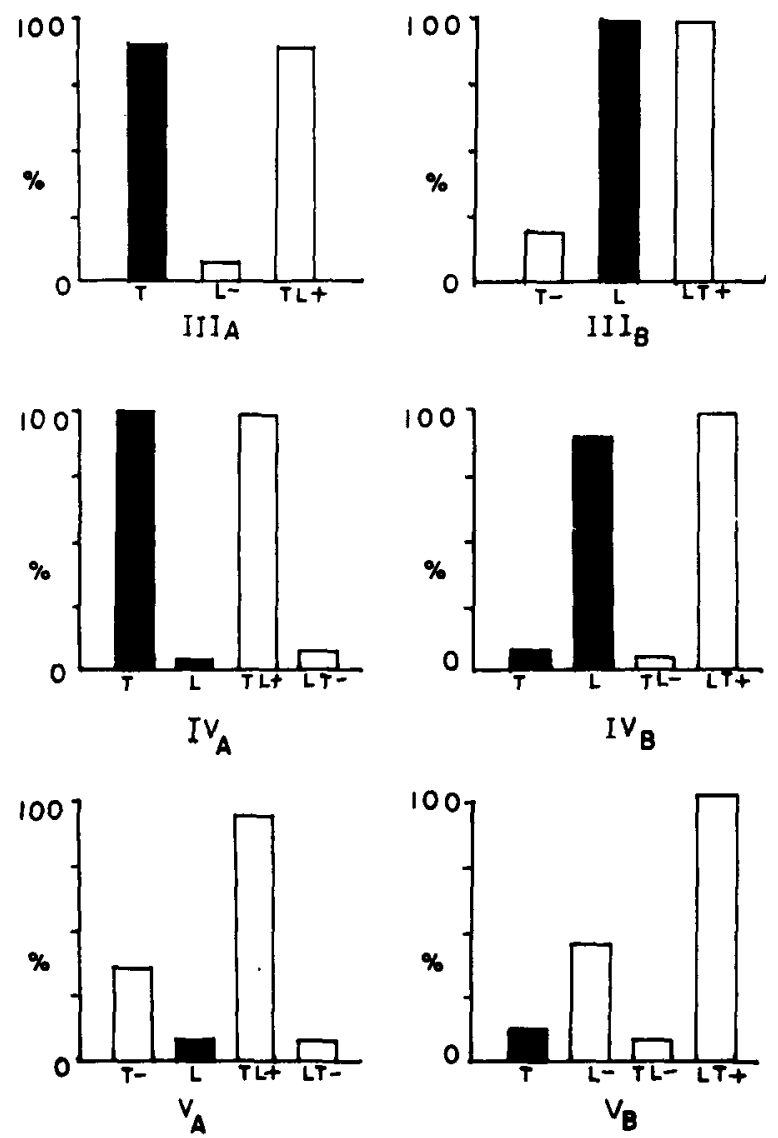

Figure 2. Percent responding during probe test trials with the various stimuli separately or in temporal compound. The left and right panels separate the counterbalanced groups to indicate control by the tone (T) and the light (L) as S1 and S2, and vice versa. The open bars represent conditions that obtained during training, and the filled bars represent generalization test conditions.

tion. Of principal significance is the fact that rats can indeed form a true temporal compound discrimination in the sense of reduced responding in the presence of either element separately or both elements presented in the reverse order. That this is a difficult accomplishment is attested to by the amount of training required to display such differential performance and the lessthan-perfect level of asymptotic performance. Furthermore, somewhat simpler discriminations involving temporal compounds appeared to be controlled predominantly by the first element of the temporal compound rather than the compound itself.

Neither the original continuity theory (Spence, 1936) nor the original noncontinuity theory (Krechevsky, 1932), or their modified versions (e.g., Mackintosh, 1975; Wagner, 1969), are immediately interpretable for temporal compound conditioning. In order to cope with the type of behavior observed, it appears necessary to incorporate notions expressed in
Hullian theory more generally. Specifically, the construct of a temporally distinctive stimulus trace and the interaction of such traces as functionally distinctive stimulus events appear to be necessary. However, it is possible that differential behavior was actually being controlled by the distinctiveness of either the first or the second component, with the other component serving a secondary function. It could be contended that the first component primed the rat to respond to the second component or, alternatively, that the second component functionally extended the duration of stimulation. Although these accounts seem more complicated than the Hullian account, they should be evaluated by generalization tests involving systematic changes in one or the other component.

Accordingly, we would go somewhat further than Razran (1939) in concluding that, "as is readily obvious, this form of compound conditioning offers a greater number of factors for study and control than that of simultaneous conditioning." The first component appears to have a dominance regardless of whether the second component is informative. The systematic experimental analysis of temporal compounds has unique relevance to theoretical analyses of stimulus control of behavior.

\section{RETERENCES}

Egger, M. D., \& Miller, N. E. Secondary reinforcement in rats as a function of information value and reliability of the stimulus. Journal of Experimental Psychology, 1962, 64, 97-104.

Hull, C. L. Principles of behavior. New York: AppletonCentury-Crofts, 1943.

Krechevsky, I. "Hypotheses" in rats. Psychological Review, 1932. 52. 516.532.

LogAN, F. A. Discrete-trials DRL. Journal of the Experimental Analysis of Behavior, 1961, 4, 277-279.

Mackintosh, N. J. Psychology of animal leaming. New York: Academic Press, 1974.

Mackintosh, N. J. Theory of attention. Psychological Review, 1975, 72, 276-298.

Razran, G. H. S. Studies of configural conditioning: Historical and preliminary experimentation. Journal of General Psychology, 1939, 21, 307-330.

SPENCE, K. W. The nature of discrimination learning in animals. Psychological Review, 1936, 43, 427-449.

WAGNer, A. R. Stimulus-selection and a "modified continuity theory." In G. H. Bower \& J. T. Spence (Eds.), The psychology of learning and motivation (Vol. 3). New York: Academic Press, 1969. Pp. 1-41.

Wickens, D. D., Bonn, D. G., \& Wickens. C. D. Response strength to a compound conditioned stimulus and its elements as a function of the element inter-stimulus interval. Journal of Comparative and Physiological Psychology, 1963. 56, 727-731.

WoodBury, C. B. The learning of stimulus patterns by dogs. Journal of Comparative Psychology, 1943. 35, 29-40.

(Received for publication September 19, 1977; revision received December 30, 1977.) 